quant-ph/9908048

LA-UR-99-1157

\title{
Higher-Power Coherent and Squeezed States 円
}

\author{
Michael Martin Nieto̊ \\ Theoretical Division (MS-B285), Los Alamos National Laboratory \\ University of California \\ Los Alamos, New Mexico 87545, U.S.A. \\ and \\ Universität Ulm, Abteilung für Quantenphysik, \\ Albert-Einstein-Allee 11, D 89069 Ulm, GERMANY \\ D. Rodney Truax[ \\ Department of Chemistry \\ University of Calgary \\ Calgary, Alberta T2N 1N4, Canada
}

July 13, 2018

\begin{abstract}
A closed form expression for the higher-power coherent states (eigenstates of $a^{j}$ ) is given. The cases $j=3,4$ are discussed in detail, including the time-evolution of the probability densities. These are compared to the case $j=2$, the even- and odd-coherent states. We give the extensions to the "effective" displacement-operator, higher-power squeezed states and to the ladder-operator/minimum-uncertainty, higher-power squeezed states. The properties of all these states are discussed.
\end{abstract}

PACS: 03.65.-w, 02.20.+b, 42.50.-p

\footnotetext{
${ }^{1}$ Dedicated to Marlan Scully on he occasion of his $60 \mathrm{~h}$ birthday.

${ }^{2}$ Email: mmn@pion.lanl.gov

${ }^{3}$ Email: truax@acs.ucalgary.ca
} 


\section{Definitions of Higher-Power Coherent States}

Higher-power coherent states (HPCS) are defined by [四]- [4]

$$
\begin{gathered}
a^{j}|\alpha ; j, k\rangle=\alpha^{j}|\alpha ; j, k\rangle, \quad 0 \leq k \leq(j-1), \\
\alpha=\alpha_{1}+i \alpha_{2} \equiv \frac{x_{0}+i p_{0}}{\sqrt{2}} .
\end{gathered}
$$

This definition produces ladder-operator-type coherent states (LOCS),

A second type of coherent states, generally equivalent to the LOCS, are minimumuncertainty coherent states (MUCS). These come from considering the operators

$$
X_{j}=\frac{a^{j}+\left(a^{\dagger}\right)^{j}}{\sqrt{2}}, \quad P_{j}=\frac{a^{j}-\left(a^{\dagger}\right)^{j}}{i \sqrt{2}},
$$

with commutation relation

$$
\left[X_{j}, P_{j}\right]=i \mathcal{O}
$$

$\mathcal{O}$ being Hermitian. This implies a Heisenberg uncertainty relation

$$
\left(\Delta X_{j}\right)^{2}\left(\Delta P_{j}\right)^{2} \geq \frac{1}{4}\left|\left\langle\left[X_{j}, P_{j}\right]\right\rangle\right|^{2}=\frac{1}{4}\langle\mathcal{O}\rangle^{2}
$$

The (wave-function) states which satisfy equality in Eq. (5) are given by solutions to the equation

$$
\left[X_{j}+i B P_{j}\right] \psi_{m u}=C \psi_{m u}
$$

where

$$
B=\frac{\langle\mathcal{O}\rangle}{2\left(\Delta P_{j}\right)^{2}}=\frac{2\left(\Delta X_{j}\right)^{2}}{\langle\mathcal{O}\rangle}, \quad C=\left\langle X_{j}\right\rangle+i B\left\langle P_{j}\right\rangle
$$

These solutions, $\psi_{m u}$ comprise not only the coherent states but also some of the squeezed states (SS) for the system. (See Sec. 5 below. Remember, the CS are special-case, zero-squeezed SS.)

To restrict the $\psi_{m u}$ to the $\psi_{c s}$, one needs to add the further restriction $\Delta X_{j} / \Delta P_{j}=$ Const. Given our present overall normalizations for $X_{j}$ and $P_{j}$, this constant is unity. For the harmonic oscillator, one has that the uncertainties in $x$ and $p$ are equal. 
For general potential systems, this constant can be determined by the demand that the set of CS include the ground-state [5]. (The ground state is the quantum analogue of zero classical motion). However, here things are complicated by the fact that the HPCS have $j$ "effective" extremal states, not just the ground state. Therefore, for a given $(j, k)$, each set $|\alpha ; j, k\rangle$ does not span the Hilbert space.

To continue, observe that there is no displacement-operator coherent-state (DOCS) definition

$$
\begin{gathered}
D_{j}(\alpha)|0\rangle=|\alpha ; j, k\rangle, \\
D_{j}(\alpha)=\exp \left[\left(\alpha^{*}\right)^{j} a^{j}-\alpha^{j}\left(a^{\dagger}\right)^{j}\right],
\end{gathered}
$$

for HPCS with $j>2$. When $j>2, a^{j}$ and $\left(a^{\dagger}\right)^{j}$ do not form part of a closed algebra and $D_{j}(\alpha)$ is not defined. $\left\langle 0\left|D_{j>2}\right| 0\right\rangle$ does not converge in a power series evaluation [4, 6]. (Even for $j=2$, potential definitions do not work. See Sec. 2.)

\section{Properties of Higher-Power Coherent States}

For $(j, k)=(1,0)$, the HPCS are the ordinary coherent states.

$$
|\alpha ; 1,0\rangle=\exp \left[-\frac{1}{2}|\alpha|^{2}\right] \sum_{n=0}^{\infty} \frac{\alpha^{n}}{\sqrt{n !}}|n\rangle \rightarrow \frac{\exp \left[-\frac{1}{2}\left(x-x_{0}\right)^{2}\right] e^{i\left[p_{0} x-x_{0} p_{0}\right]}}{\pi^{1 / 4}} .
$$

(We use units $\hbar=m=\omega=1$.) Up to a phase, the three LOCS, MUCS, and DOCS definitions yield the same Eq. (10).

The even- and odd-coherent states [1, 4, 8] are HPCS with $(j, k)=(2,0)$ and $(2,1)$. The LOCS and MUCS methods both lead to

$$
\begin{gathered}
|\alpha ; 2,0\rangle=\left[\cosh |\alpha|^{2}\right]^{-1 / 2} \sum_{n=0}^{\infty} \frac{\alpha^{2 n}}{\sqrt{(2 n) !}}|2 n\rangle \rightarrow \psi_{+}(x), \\
|\alpha ; 2,1\rangle=\left[\sinh |\alpha|^{2}\right]^{-1 / 2} \sum_{n=0}^{\infty} \frac{\alpha^{2 n+1}}{\sqrt{(2 n+1) !}}|2 n+1\rangle \rightarrow \psi_{-}(x) . \\
\psi_{ \pm}(x)=\frac{e^{-i 2 x_{0} p_{0}}\left[\exp \left[-\frac{1}{2}\left(x-x_{0}\right)^{2}\right] e^{i p_{0} x} \pm \exp \left[-\frac{1}{2}\left(x+x_{0}\right)^{2}\right] e^{-i p_{0} x}\right]}{\left.2^{1 / 2} \pi^{1 / 4}\left[1 \pm \exp \left[-\left(x_{0}^{2}+p_{0}^{2}\right)\right]\right)\right]^{1 / 2}} .
\end{gathered}
$$


The wave packets of these states are two Gaussians, at positions $\pi$ apart in the phasespace circle. The even states are composed of $n=0,2,4, \ldots$ number states. These Gaussians, when they interfere, have a maximum central peak [7, 8]. (See Figure 1.) The odd states are composed of $n=1,3,5, \ldots$ number states. When the odd Gaussians interfere there is a central minimum and two slightly smaller peaks on each side [0, 8]. (See Figure 2.)

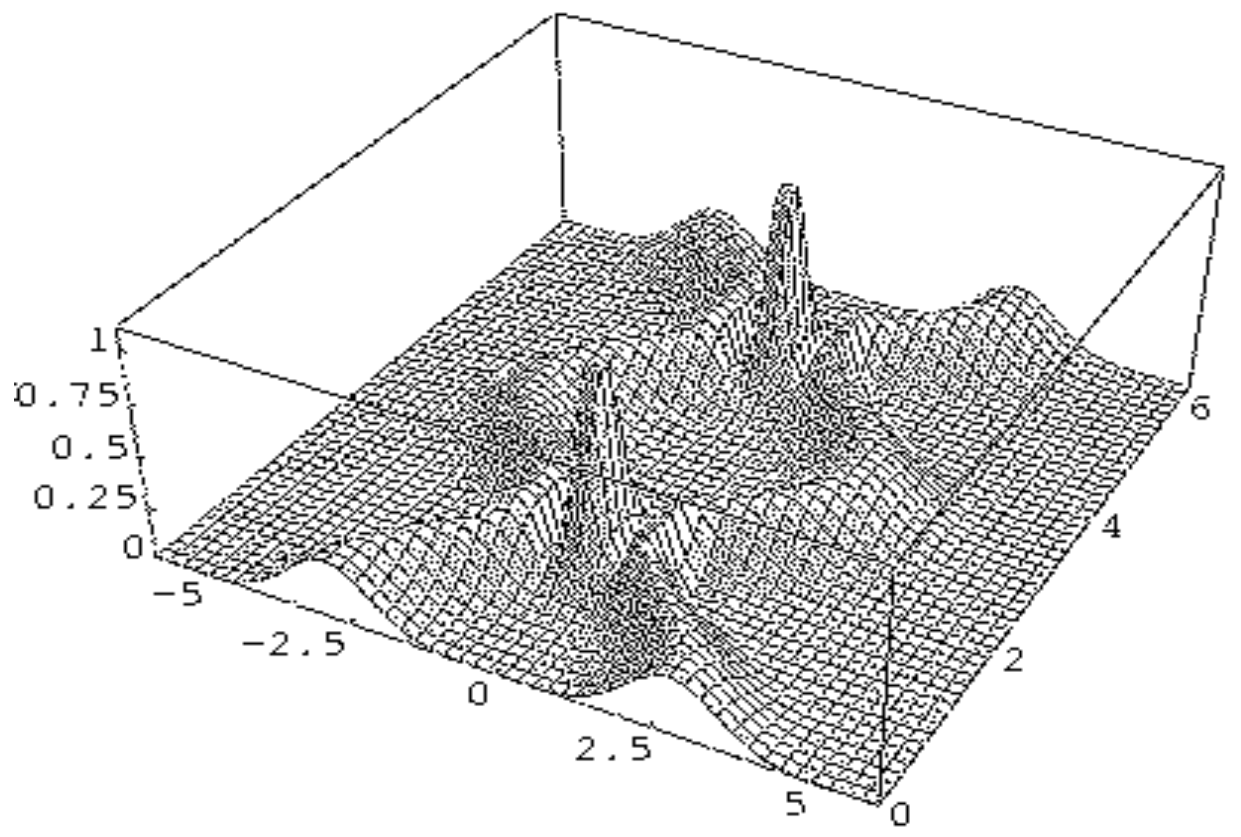

Figure 1: The time evolution of the even-coherent state $\rho_{(2,0)}(x, t)$ for the initial conditions $x_{0}=2^{3 / 2}$ and $p_{0}=0$.

As stated above, the DOCS method does not work for $j>2$ and already has problems even for the $\mathrm{j}=2$ case. One might think a "viable" displacement operator could be given by the form of the ordinary squeeze operator $S\left(z=2 \alpha^{2}\right)$ of Eq. (57) below:

$$
D_{2}(\alpha)=\exp \left[\left(\alpha^{*}\right)^{2} a^{2}-\alpha^{2}\left(a^{\dagger}\right)^{2}\right]
$$

Applying this operator to the true extremal state $|0\rangle$ does produce an even state. To obtain an odd state, you have to apply $S$ by hand to the state $|1\rangle$, which is outside 


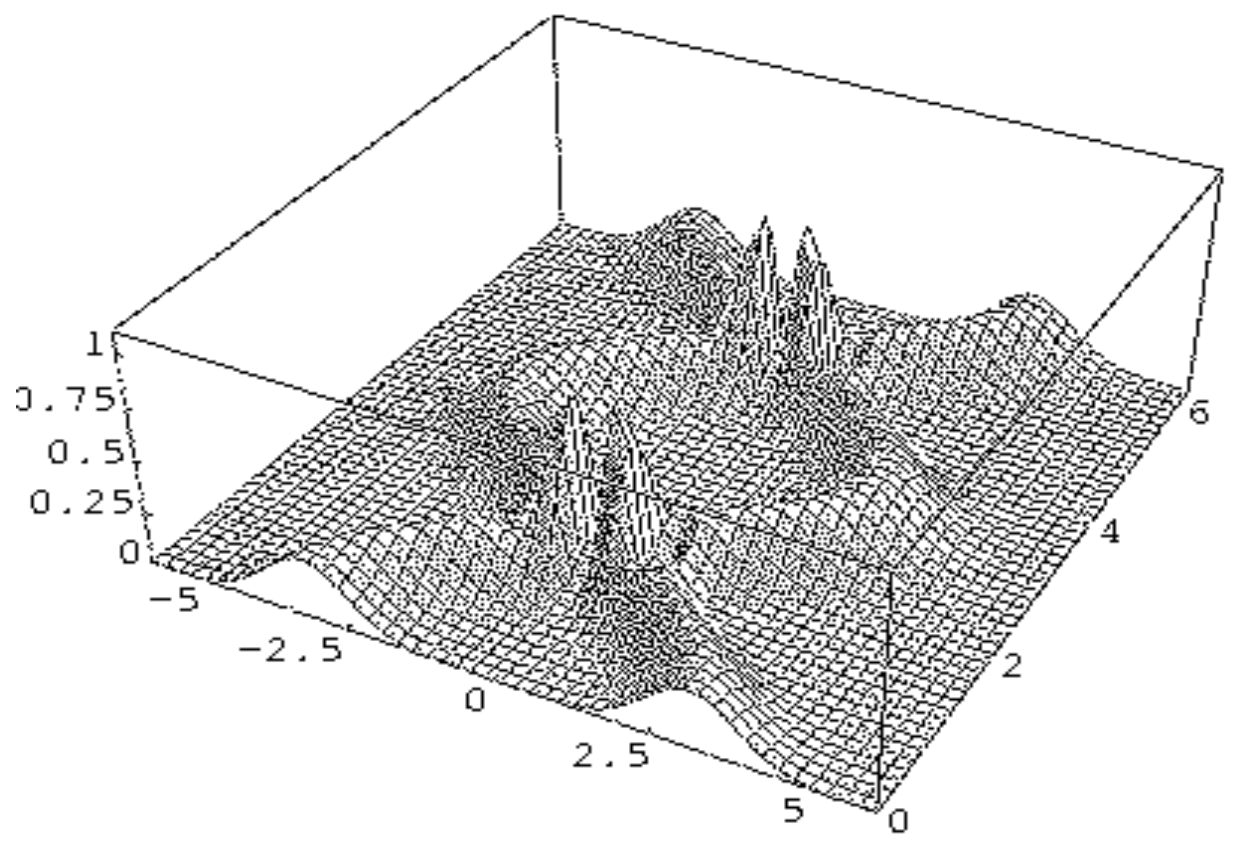

Figure 2: The time evolution of the odd-coherent state $\rho_{(2,1)}(x, t)$ for the initial conditions $x_{0}=\sqrt{10}$ and $p_{0}=0$.

the usual method. However, the even and odd states so produced are not the evenand odd-CS. Rather, they are the squeezed (but not displaced) number states with $n=(0,1)[9]$. One can also devise "effective" displacement operators [1]

$$
D_{ \pm}(\alpha)=N_{ \pm}\left[D_{1}(\alpha) \pm D_{1}(-\alpha)\right], \quad D_{ \pm}(\alpha)|0\rangle=\left|\alpha ; 2, \frac{1}{2} \mp \frac{1}{2}\right\rangle .
$$

But these operators are not unitary: $D_{ \pm}(\alpha) D_{ \pm}^{\dagger}(\alpha) \neq I$.

From these $j=1,2$ examples, it is clear what occurs in general for higher- $j$ HPCS of the LOCS/MUCS variety. Given $j$ and $\alpha$, there are $j$ states, labeled by $(j, k)$ with $0 \leq k \leq(j-1)$. They are separately composed of the number states: $\{0, j, 2 j, \ldots\}$, $\{1,(j+1),(2 j+1), \ldots\},\{2,(j+2),(2 j+2), \ldots\}, \ldots$, and $\{(j-1),(2 j-1),(3 j-1), \ldots\}$. In wave-function space, these $j$ states all each contain $j$ Gaussians, separated by $2 \pi / j$ in the phase-space circle. The relative phases among the $j$ Gaussians in each state are adjusted so that the $(j, k)$-power states are mutually orthogonal.

Using higher-order Hermite generating function techniques [3], one can use Eq. 
(1) to obtain closed-form expressions for these higher-power states. Specifically, the orthonormal states are

$$
\begin{aligned}
|\alpha ; j, k\rangle & =\mathcal{S}^{-1 / 2}\left(j, k,|\alpha|^{2}\right) \sum_{n=0}^{\infty} \frac{\alpha^{j n+k}}{\sqrt{(j n+k) !}}|j n+k\rangle \\
& \rightarrow \psi_{(j, k)}(x)=\frac{\exp \left[-\frac{1}{2} x^{2}\right] G(j, k, x, \alpha / \sqrt{2})}{\pi^{1 / 4} \mathcal{S}^{1 / 2}\left(j, k,|\alpha|^{2}\right)}
\end{aligned}
$$

In the above, $\mathcal{S}$ is the sum

$$
\mathcal{S}(j, k, z)=\sum_{n=0}^{\infty} \frac{z^{j n+k}}{(j n+k) !}=\frac{1}{j} \sum_{l=1}^{j} \frac{\exp \left[z e^{i 2 \pi l / j}\right]}{\left[e^{i 2 \pi l / j}\right]^{k}},
$$

and $G$ is the higher-order Hermite generating function [3]

$$
G(j, k, x, z)=\sum_{n=0}^{\infty} \frac{z^{j n+k} H_{j n+k}(x)}{(j n+k) !}=\frac{1}{j} \sum_{l=1}^{j} \frac{\exp \left[-z^{2} e^{i 4 \pi l / j}\right] \exp \left[2 x z e^{i 2 \pi l / j}\right]}{\left[e^{i 2 \pi l / j}\right]^{k}} .
$$

\section{Special-Case Higher-Power Coherent States}

\subsection{The Case $j=3$}

Let us now look at the $j=3$ states in detail. First define

$$
\begin{aligned}
A & =\frac{x_{0}^{2}+p_{0}^{2}}{2}, \\
N_{(3,0)} & =\left[1+2 \cos \left(\frac{\sqrt{3}}{2} A\right)\right] \\
N_{(3,1)} & =\left[1-\left(\cos \left(\frac{\sqrt{3}}{2} A\right)-\sin \left(\frac{\sqrt{3}}{2} A\right)\right) e^{-3 A / 2}\right], \\
N_{(3,2)} & =\left[1-\left(\cos \left(\frac{\sqrt{3}}{2} A\right)+\sin \left(\frac{\sqrt{3}}{2} A\right)\right) e^{-3 A / 2}\right], \\
Y_{1} & =\exp \left[-\frac{1}{2}\left[x+\left(\frac{1}{2} x_{0}+\frac{\sqrt{3}}{2} p_{0}\right)\right]^{2}\right] e^{i\left[x\left(\frac{\sqrt{3}}{2} x_{0}-\frac{1}{2} p_{0}\right)+\frac{\sqrt{3}}{8}\left(x_{0}^{2}-p_{0}^{2}\right)+\frac{1}{4} x_{0} p_{0}\right]}, \\
Y_{2} & =\exp \left[-\frac{1}{2}\left[x+\left(\frac{1}{2} x_{0}-\frac{\sqrt{3}}{2} p_{0}\right)\right]^{2}\right] e^{i\left[-x\left(\frac{\sqrt{3}}{2} x_{0}+\frac{1}{2} p_{0}\right)+\frac{\sqrt{3}}{8}\left(x_{0}^{2}-p_{0}^{2}\right)+\frac{1}{4} x_{0} p_{0}\right]}, \\
Y_{3} & =\exp \left[-\frac{1}{2}\left[x-x_{0}\right]^{2}\right] e^{i\left[x p_{0}-\frac{1}{2} x_{0} p_{0}\right]},
\end{aligned}
$$

The three, orthonormal, 3-power coherent states are then

$$
\psi_{(3,0)}(x)=\frac{Y_{1}+Y_{2}+Y_{3}}{3^{1 / 2} \pi^{1 / 4} N_{(3,0)}^{1 / 2}}
$$




$$
\begin{aligned}
& \psi_{(3,1)}(x)=\frac{\left[-\frac{1}{2}-i \frac{\sqrt{3}}{2}\right] Y_{1}+\left[-\frac{1}{2}+i \frac{\sqrt{3}}{2}\right] Y_{2}+Y_{3}}{3^{1 / 2} \pi^{1 / 4} N_{(3,1)}^{1 / 2}} \\
& \psi_{(3,2)}(x)=\frac{\left[-\frac{1}{2}+i \frac{\sqrt{3}}{2}\right] Y_{1}+\left[-\frac{1}{2}-i \frac{\sqrt{3}}{2}\right] Y_{2}+Y_{3}}{3^{1 / 2} \pi^{1 / 4} N_{(3,2)}^{1 / 2}}
\end{aligned}
$$

If we define the angles,

$$
\begin{aligned}
& \phi_{1,2}=x\left(\sqrt{3} x_{0}\right)+\frac{\sqrt{3}}{4}\left(x_{0}^{2}-p_{0}^{2}\right), \\
& \phi_{1,3}=x\left(\frac{\sqrt{3}}{2} x_{0}-\frac{3}{2} p_{0}\right)+\frac{\sqrt{3}}{8}\left(x_{0}^{2}-p_{0}^{2}\right)+\frac{3}{4} x_{0} p_{0}, \\
& \phi_{2,3}=x\left(\frac{\sqrt{3}}{2} x_{0}+\frac{3}{2} p_{0}\right)-\frac{\sqrt{3}}{8}\left(x_{0}^{2}-p_{0}^{2}\right)+\frac{3}{4} x_{0} p_{0},
\end{aligned}
$$

then the three probability densities

$$
\rho_{(3, k)}(x)=\psi_{(3, k)}^{*}(x) \psi_{(3, k)}(x)
$$

are

$$
\begin{aligned}
\rho_{(3,0)}(x)= & \frac{1}{3 \pi^{1 / 2} N_{(3,0)}}\left\{\left|Y_{1}^{2}\right|+\left|Y_{2}^{2}\right|+\left|Y_{3}^{2}\right|+2 \cos \phi_{1,2}\left|Y_{1} Y_{2}\right|\right. \\
& \left.+2 \cos \phi_{1,3}\left|Y_{1} Y_{3}\right|+2 \cos \phi_{2,3}\left|Y_{2} Y_{3}\right|\right\}, \\
\rho_{(3,1)}(x)= & \frac{1}{3 \pi^{1 / 2} N_{(3,1)}}\left\{\left|Y_{1}^{2}\right|+\left|Y_{2}^{2}\right|+\left|Y_{3}^{2}\right|-\left(\cos \phi_{1,2}+\sqrt{3} \sin \phi_{1,2}\right)\left|Y_{1} Y_{2}\right|\right. \\
& \left.-\left(\cos \phi_{1,3}-\sqrt{3} \sin \phi_{1,3}\right)\left|Y_{1} Y_{3}\right|-\left(\cos \phi_{2,3}+\sqrt{3} \sin \phi_{2,3}\right)\left|Y_{2} Y_{3}\right|\right\}, \\
\rho_{(3,2)}(x)= & \frac{1}{3 \pi^{1 / 2} N_{(3,2)}}\left\{\left|Y_{1}^{2}\right|+\left|Y_{2}^{2}\right|+\left|Y_{3}^{2}\right|-\left(\cos \phi_{1,2}-\sqrt{3} \sin \phi_{1,2}\right)\left|Y_{1} Y_{2}\right|\right. \\
& \left.-\left(\cos \phi_{1,3}+\sqrt{3} \sin \phi_{1,3}\right)\left|Y_{1} Y_{3}\right|-\left(\cos \phi_{2,3}-\sqrt{3} \sin \phi_{2,3}\right)\left|Y_{2} Y_{3}\right|\right\} .
\end{aligned}
$$

Because we are working in an harmonic-oscillator system, time-dependence is achieved by taking

$$
x_{0} \rightarrow x_{0} \cos t+p_{0} \sin t, \quad p_{0} \rightarrow p_{0} \cos t-x_{0} \sin t .
$$

in $\psi_{(3, k)}(x)$ and $\rho_{(3, k)}(x)$. 
In Figures 3 , 因, and 0 we show the time-evolution of $\rho_{(3, k)}(x, t)$ for $k=0,1,2$, with initial conditions $x_{0}=0$ and $p_{0}=10$. With these initial conditions, the interference patters are more peaked than in Figs. 1 and 2. All three figures show interference patterns that do not exactly look like either an even or odd interference. This is not surprising, since the basic phase angle is $2 \pi / 3$. However, Figure $3[(j, k)=(3,0)]$ and Figure $1[(j, k)=(3,1)]$ have interferences closer to odd ones, while Figure 5 $[(j, k)=(3,2)]$ has interferences closer to even ones.

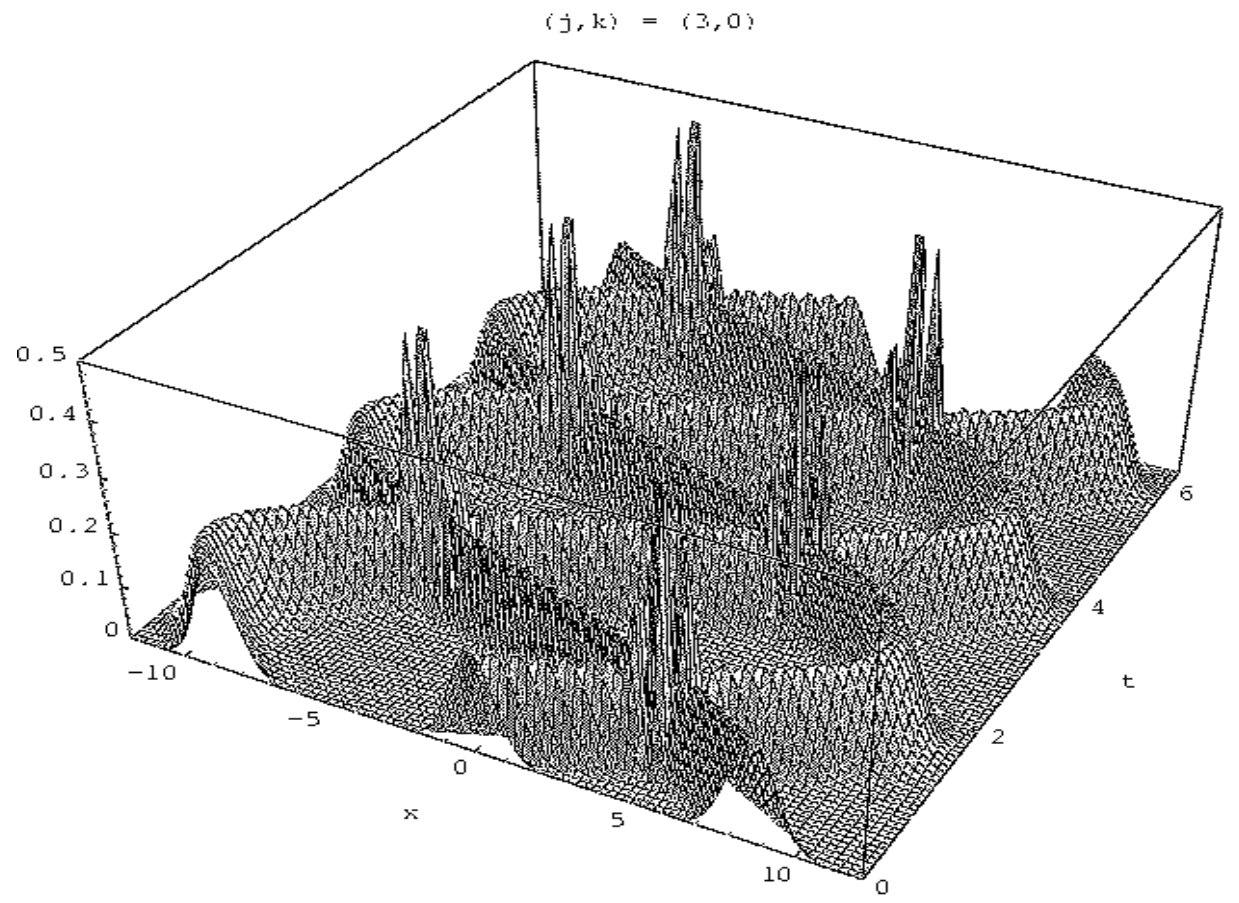

Figure 3: The time evolution of $\rho_{(3,0)}(x, t)$ for the initial conditions $x_{0}=0$ and $p_{0}=10$. 


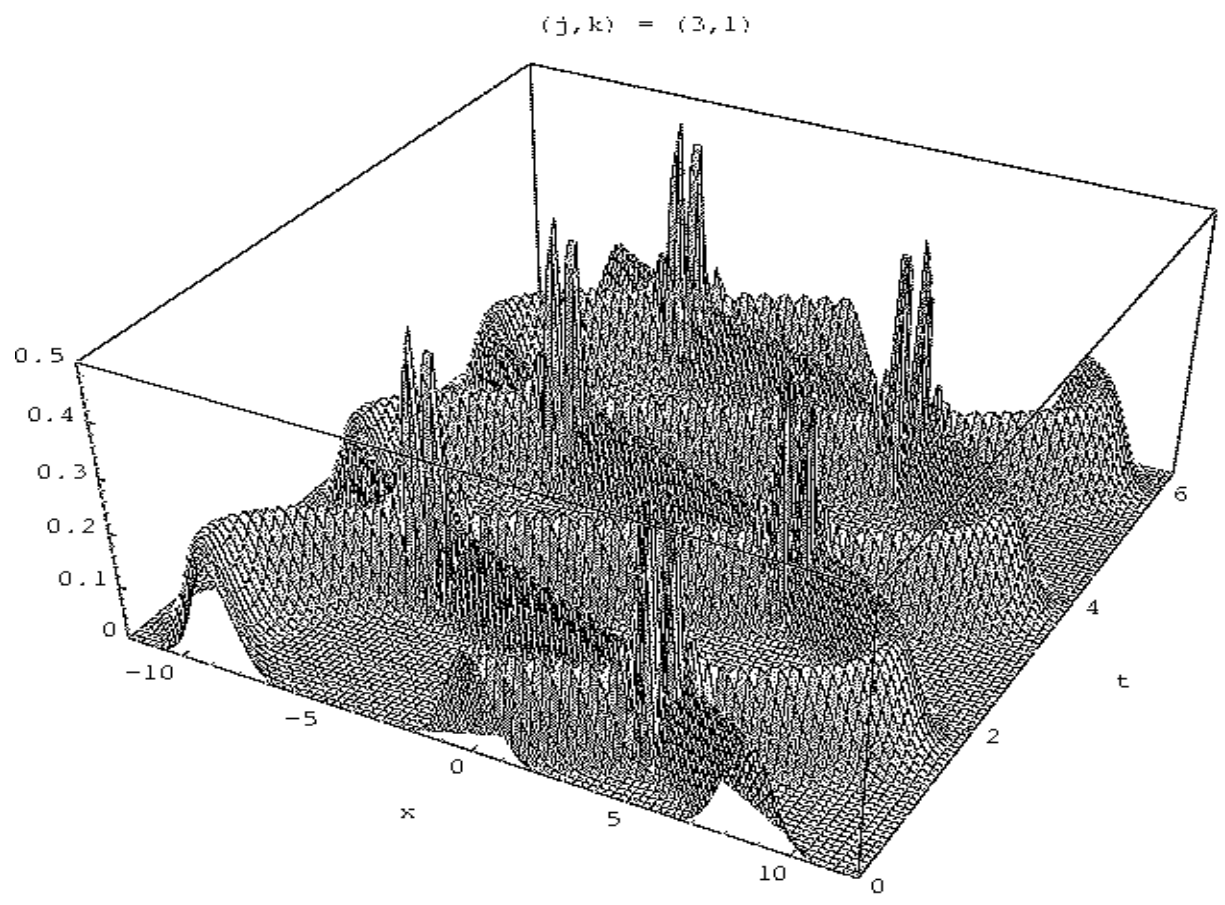

Figure 4: The time evolution of $\rho_{(3,1)}(x, t)$ for the initial conditions $x_{0}=0$ and $p_{0}=10$.

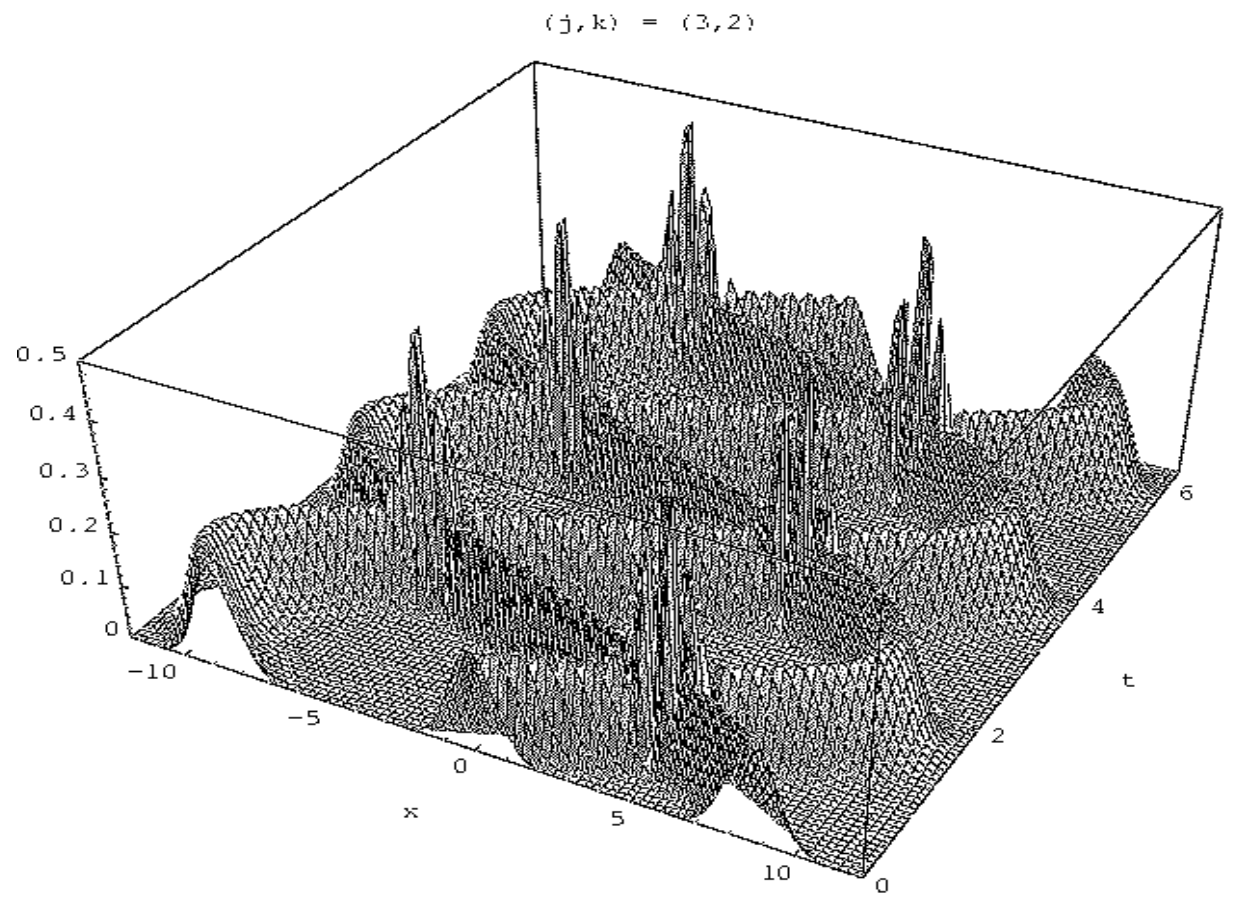

Figure 5: The time evolution of $\rho_{(3,2)}(x, t)$ for the initial conditions $x_{0}=0$ and $p_{0}=10$. 


\subsection{The Case $j=4$}

Now consider the $j=4$ states. Define

$$
\begin{aligned}
N_{(4,0)} & =\cosh A+\cos A, \quad N_{(4,1)}=\sinh A+\sin A \\
N_{(4,2)} & =\cosh A-\cos A, \quad N_{(4,3)}=\sinh A-\sin A \\
Z_{1} & =\exp \left[-\frac{1}{2}\left(x-x_{0}\right)^{2}\right] e^{i p_{0}\left(x-x_{0}\right)}, \\
Z_{2} & =\exp \left[-\frac{1}{2}\left(x-p_{0}\right)^{2}\right] e^{-i x_{0}\left(x-p_{0}\right)}, \\
Z_{3} & =\exp \left[-\frac{1}{2}\left(x+x_{0}\right)^{2}\right] e^{-i p_{0}\left(x+x_{0}\right)}, \\
Z_{4} & =\exp \left[-\frac{1}{2}\left(x+p_{0}\right)^{2}\right] e^{i x_{0}\left(x+p_{0}\right)}, \\
\theta_{1,2} & =x\left(x_{0}+p_{0}\right)-p_{0} x_{0}, \quad \theta_{1,3}=2 x p_{0}, \quad \theta_{1,4}=x\left(p_{0}-x_{0}\right)-x_{0} p_{0}, \\
\theta_{2,3} & =x\left(p_{0}-x_{0}\right)+x_{0} p_{0}, \quad \theta_{2,4}=2 x x_{0}, \quad \theta_{3,4}=-x\left(x_{0}+p_{0}\right)-x_{0} p_{0} .
\end{aligned}
$$

The states $\psi_{(4, k)}(x)$ are then

$$
\begin{aligned}
& \psi_{(4,0)}(x)=\frac{e^{A / 2}}{2^{3 / 2} \pi^{1 / 4} N_{(4,0)}^{1 / 2}}\left[Z_{1}+Z_{2}+Z_{3}+Z_{4}\right], \\
& \psi_{(4,1)}(x)=\frac{e^{A / 2}}{2^{3 / 2} \pi^{1 / 4} N_{(4,1)}^{1 / 2}}\left[Z_{1}+i Z_{2}-Z_{3}-i Z_{4}\right], \\
& \psi_{(4,2)}(x)=\frac{e^{A / 2}}{2^{3 / 2} \pi^{1 / 4} N_{(4,2)}^{1 / 2}}\left[Z_{1}-Z_{2}+Z_{3}-Z_{4}\right], \\
& \psi_{(4,3)}(x)=\frac{e^{A / 2}}{2^{3 / 2} \pi^{1 / 4} N_{(4,3)}^{1 / 2}}\left[Z_{1}-i Z_{2}-Z_{3}+i Z_{4}\right] .
\end{aligned}
$$

The probability densities $\rho_{(4, k)}(x)$ are

$$
\begin{aligned}
\rho_{(4,0)}(x)= & \frac{e^{A}}{8 \pi^{1 / 2} N_{(4,0)}}\left\{\left|Z_{1}\right|^{2}+\left|Z_{2}\right|^{2}+\left|Z_{3}\right|^{2}+\left|Z_{4}\right|^{2}\right. \\
& +2 \cos \theta_{1,2}\left|Z_{1} Z_{2}\right|+2 \cos \theta_{1,3}\left|Z_{1} Z_{3}\right|+2 \cos \theta_{1,4}\left|Z_{1} Z_{4}\right| \\
& \left.+2 \cos \theta_{2,3}\left|Z_{2} Z_{3}\right|+2 \cos \theta_{2,4}\left|Z_{2} Z_{4}\right|+2 \cos \theta_{3,4}\left|Z_{3} Z_{4}\right|\right\}, \\
\rho_{(4,1)}(x)= & \frac{e^{A}}{8 \pi^{1 / 2} N_{(4,1)}}\left\{\left|Z_{1}\right|^{2}+\left|Z_{2}\right|^{2}+\left|Z_{3}\right|^{2}+\left|Z_{4}\right|^{2}\right.
\end{aligned}
$$




$$
\begin{aligned}
& +2 \sin \theta_{1,2}\left|Z_{1} Z_{2}\right|-2 \cos \theta_{1,3}\left|Z_{1} Z_{3}\right|-2 \sin \theta_{1,4}\left|Z_{1} Z_{4}\right| \\
& \left.+2 \sin \theta_{2,3}\left|Z_{2} Z_{3}\right|-2 \cos \theta_{2,4}\left|Z_{2} Z_{4}\right|+2 \sin \theta_{3,4}\left|Z_{3} Z_{4}\right|\right\}, \\
\rho_{(4,2)}(x)= & \frac{e^{A}}{8 \pi^{1 / 2} N_{(4,2)}}\left\{\left|Z_{1}\right|^{2}+\left|Z_{2}\right|^{2}+\left|Z_{3}\right|^{2}+\left|Z_{4}\right|^{2}\right. \\
& -2 \cos \theta_{1,2}\left|Z_{1} Z_{2}\right|+2 \cos \theta_{1,3}\left|Z_{1} Z_{3}\right|-2 \cos \theta_{1,4}\left|Z_{1} Z_{4}\right| \\
\rho_{(4,3)}(x)= & \frac{\left.-2 \cos \theta_{2,3}\left|Z_{2} Z_{3}\right|+2 \cos \theta_{2,4}\left|Z_{2} Z_{4}\right|-2 \cos \theta_{3,4}\left|Z_{3} Z_{4}\right|\right\},}{8 \pi^{1 / 2} N_{(4,3)}}\left\{\left|Z_{1}\right|^{2}+\left|Z_{2}\right|^{2}+\left|Z_{3}\right|^{2}+\left|Z_{4}\right|^{2}\right. \\
& -2 \sin \theta_{1,2}\left|Z_{1} Z_{2}\right|-2 \cos \theta_{1,3}\left|Z_{1} Z_{3}\right|+2 \sin \theta_{1,4}\left|Z_{1} Z_{4}\right| \\
& \left.-2 \sin \theta_{2,3}\left|Z_{2} Z_{3}\right|-2 \cos \theta_{2,4}\left|Z_{2} Z_{4}\right|-2 \sin \theta_{3,4}\left|Z_{3} Z_{4}\right|\right\} .
\end{aligned}
$$

In Figures 6 to 9, we show the time-evolution of $\rho_{(4, k)}(x, t)$ for $k=0,1,2,3$, with initial conditions $x_{0}=0$ and $p_{0}=10$. Again, with these initial conditions the interference patters are more peaked than in Figs. 11 and 2. In this example, all four figures show interference patterns that resemble either even or odd interferences. This is still not surprising since this time the basic phase angle is $\pi / 2$. Figure 6 $[(j, k)=(4,0)]$ has interferences that appear odd. Figure $0[(j, k)=(4,1)]$ has central interferences that are even, but outer interferences that are closer to odd. Figure $8[(j, k)=(4,2)]$ has central interferences that are odd, but outer interferences that are even. Finally, Figure $9[(j, k)=(4,3)]$ has interferences that all appear even. 


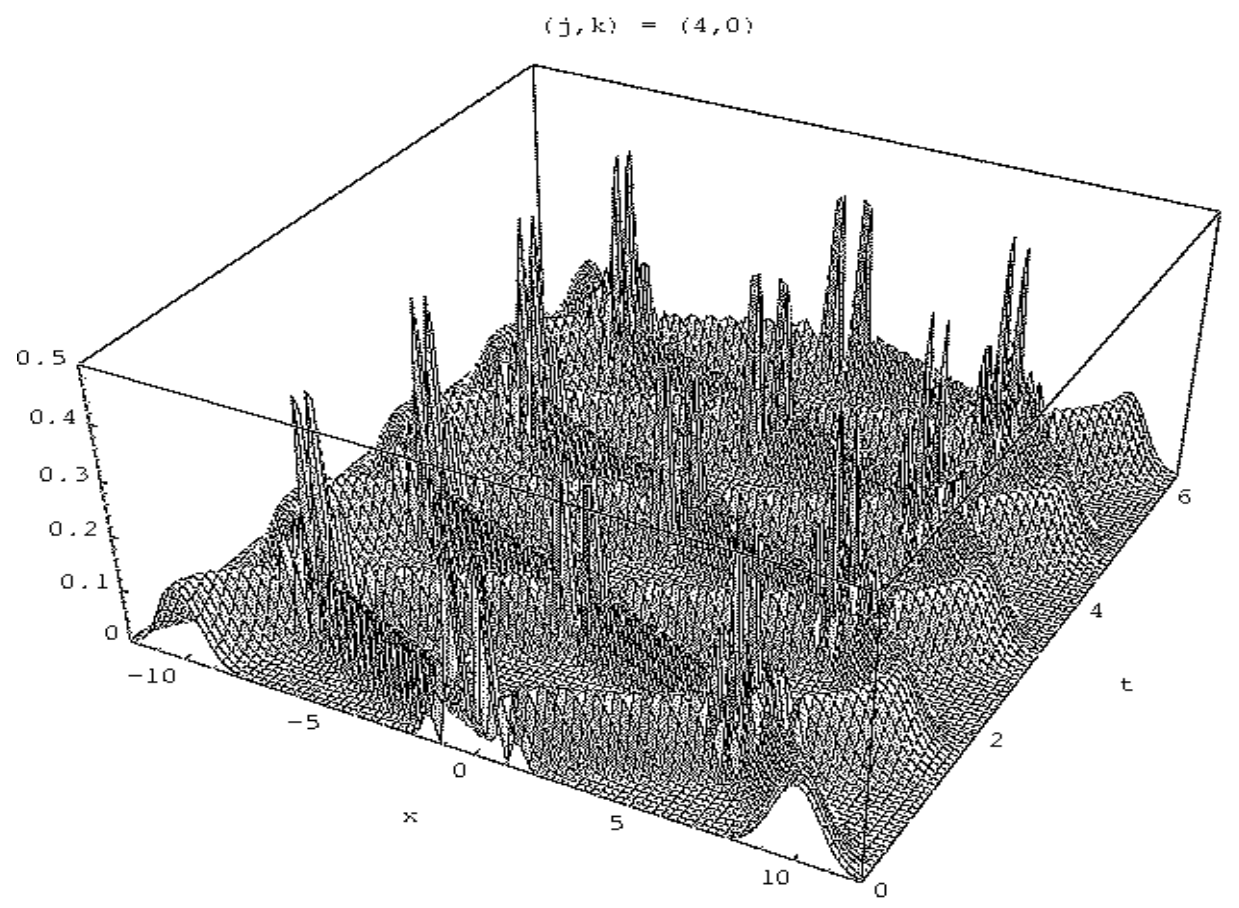

Figure 6: The time evolution of $\rho_{(4,0)}(x, t)$ for the initial conditions $x_{0}=0$ and $p_{0}=10$.

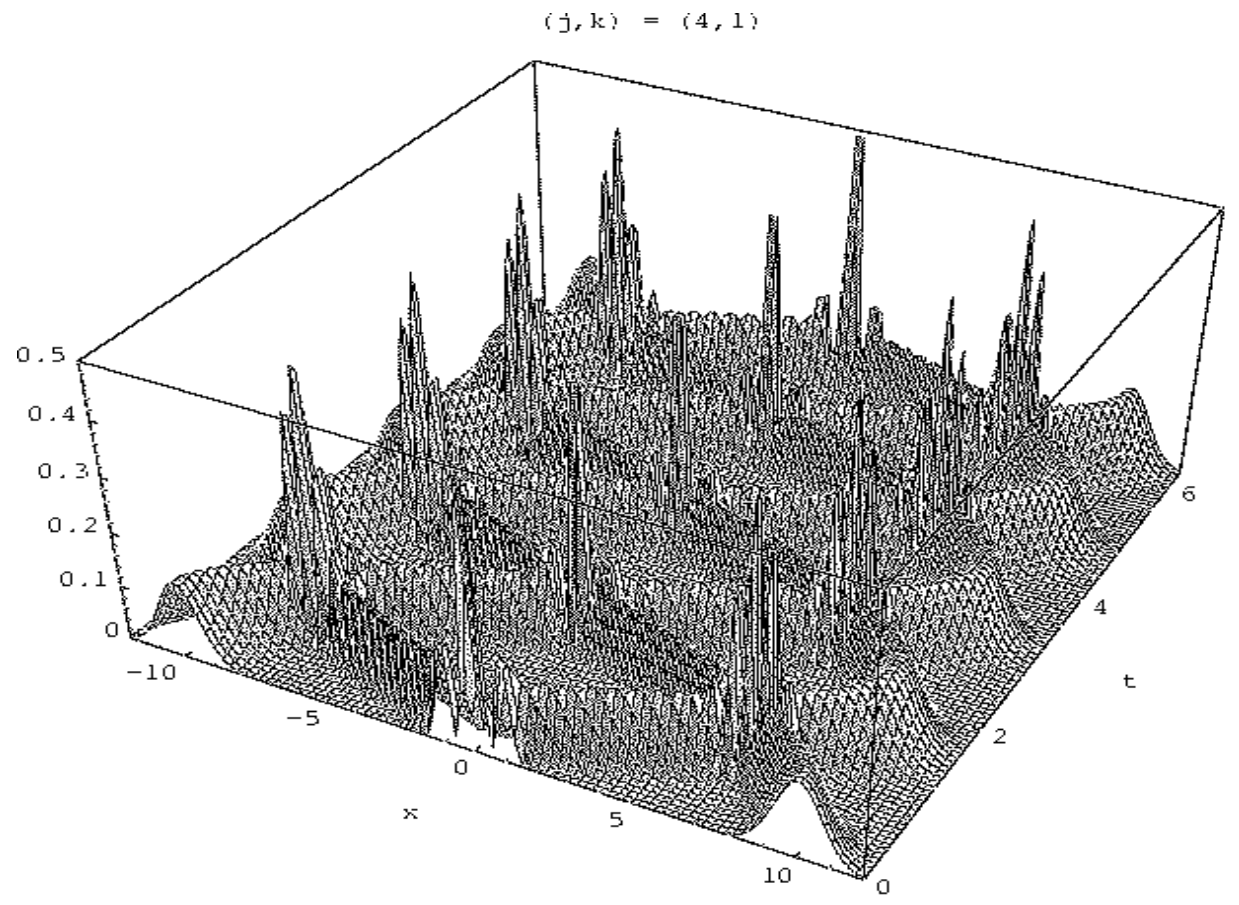

Figure 7: The time evolution of $\rho_{(4,1)}(x, t)$ for the initial conditions $x_{0}=0$ and $p_{0}=10$. 


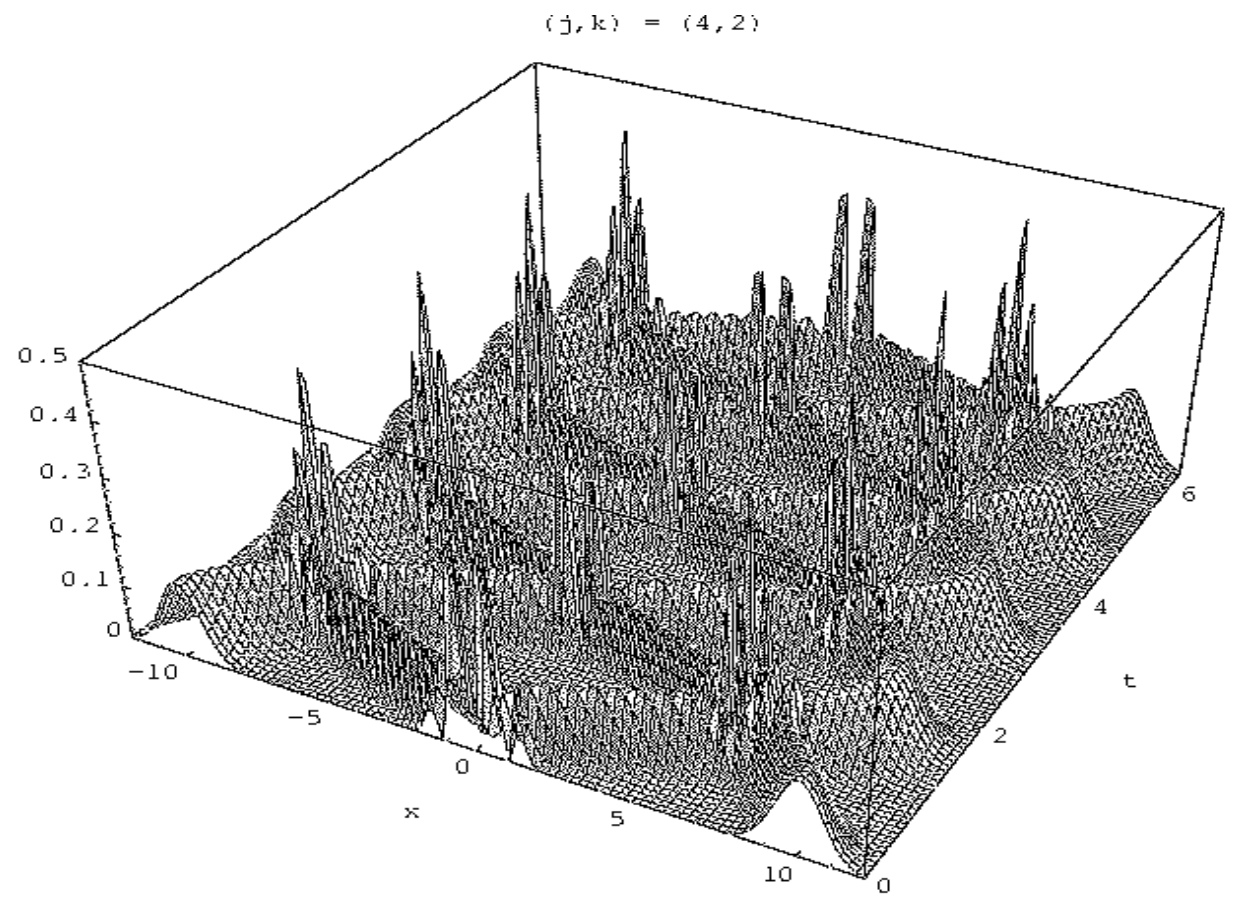

Figure 8: The time evolution of $\rho_{(4,2)}(x, t)$ for the initial conditions $x_{0}=0$ and $p_{0}=10$.

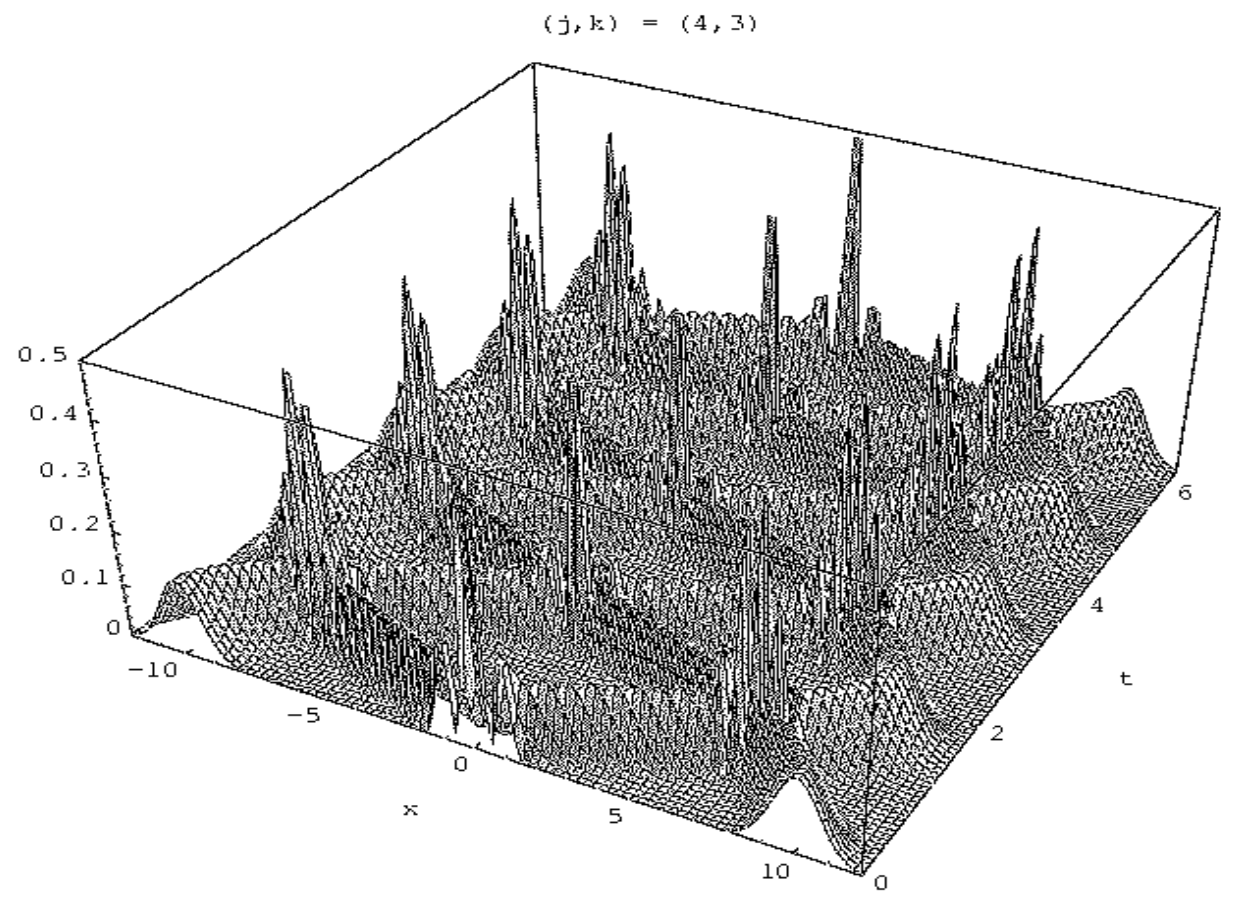

Figure 9: The time evolution of $\rho_{(4,3)}(x, t)$ for the initial conditions $x_{0}=0$ and $p_{0}=10$. 


\section{4 "Effective" Displacement-Operator Squeezed States.}

The DOSS for the harmonic oscillator are well-known:

$$
\begin{gathered}
S(z) D(\alpha)|0\rangle=|\beta\rangle, \quad S(z) a S^{-1}(z)|\beta\rangle=\left(\mu a+\nu a^{\dagger}\right)|\beta\rangle=\beta|\beta\rangle, \\
\mu=\cosh r, \quad \nu=-e^{i \phi} \sinh r, \quad \beta=\left[(\mu+\nu) x_{0}+i(\mu-\nu) p_{0}\right] / \sqrt{2}, \\
|\beta\rangle \rightarrow\left[\frac{(\mu+\nu)}{\pi^{1 / 2}(\mu-\nu)}\right]^{1 / 2} \exp \left[-\frac{\left(x-x_{0}\right)^{2}}{2} \frac{(\mu+\nu)}{(\mu-\nu)}+i p_{0} x\right],
\end{gathered}
$$

where the $s u(1,1)$ squeeze operator is

$$
S(z)=\exp \left[z a^{\dagger 2} / 2-z^{*} a^{2} / 2\right], \quad z=r e^{i \phi},
$$

When one tries to extend this definition to high- $j$ HPSS, one runs into problems. Similar to what was said for HPCS with $j>2$, there is no group-theoretic method to define higher-power squeeze operators for $j>1$ [4, 6]. For $j>1, a^{2 j}$ and $\left(a^{\dagger}\right)^{2 j}$ do not form part of a closed algebra.

However, there is an "effective" displacement-operator ansatz that can be used [6]. First, one applies the ordinary squeeze operator, $S(z)$, to $a^{j}$. By then inserting $I=S^{-1}(z) S(z)$ after each $a$, one finds

$$
\left[S(z) a S^{-1}(z)\right]^{j}|\bar{\beta} ; j, k\rangle=\bar{\beta}^{j}|\bar{\beta} ; j, k\rangle, \quad|\bar{\beta} ; j, k\rangle=S(z)|\alpha ; j, k\rangle .
$$

These squeezed states $|\bar{\beta} ; j, k\rangle$ are eigenvalues of the operator

$$
\left[S(z) a S^{-1}(z)\right]^{j}=\left(\bar{\mu} a+\bar{\nu} a^{\dagger}\right)^{j}, \quad|\bar{\mu}|^{2}-|\bar{\nu}|^{2}=1 .
$$

Similar to before, these states are a subset of those states which satisfy equality of the Heisenberg uncertainty relation

$$
\left(\Delta \mathcal{X}_{j}\right)^{2}\left(\Delta \mathcal{P}_{j}\right)^{2} \geq \frac{1}{4}\left|\left\langle\left[\mathcal{X}_{j}, \mathcal{P}_{j}\right]\right\rangle\right|^{2}
$$

where

$$
\mathcal{X}_{j}=\frac{\left(\bar{\mu} a+\bar{\nu} a^{\dagger}\right)^{j}+\left[\left(\bar{\mu} a+\bar{\nu} a^{\dagger}\right)^{j}\right]^{\dagger}}{\sqrt{2}}, \quad \mathcal{P}_{j}=\frac{\left(\bar{\mu} a+\bar{\nu} a^{\dagger}\right)^{j}-\left[\left(\bar{\mu} a+\bar{\nu} a^{\dagger}\right)^{j}\right]^{\dagger}}{i \sqrt{2}} .
$$


[See. Eq. (6)]. The subset is defined by the additional restriction that $\Delta \mathcal{X}_{j} / \Delta \mathcal{P}_{j}=1$.

The $j$ Gaussians of a $(j, k)$ HPCS are thereby transformed into $j$ squeezed Gaussians, similar to the ordinary squeezed-state Gaussian of Eq. (56). Then, for example, the even- and odd-like interferences in Figures 39 will become even- and odd-like squeezed interferences. They will be similar to those shown in Ref. [8] for the even and odd squeezed states with $z$ real (with $s=e^{r}$ ),

$$
\psi_{s s \pm}=\left[\pi^{1 / 2} 2 s\left(1 \pm e^{-x_{0}^{2} / s^{2}-p_{0}^{2} s^{2}}\right)\right]^{-1 / 2}\left[e^{-\left(x-x_{0}\right)^{2} /\left(2 s^{2}\right)+i p_{0} x} \pm e^{-\left(x+x_{0}\right)^{2} /\left(2 s^{2}\right)-i p_{0} x}\right] .
$$

\section{Ladder-Operator/Minimum-Uncertainty Squeezed States.}

When $j>1$, the ladder-operator (LO)/minimum-uncertainty (MU) method has its own problem. However, it is a technical problem, rather than one of principle. To calculate the squeezed states in closed form, becomes increasingly difficult as $j$ increases.

This LO method has as its defining equations

$$
\begin{aligned}
{\left[\mu^{j} a^{j}+\nu^{j} a^{\dagger j}\right]|\beta ; j, k\rangle } & =\beta^{j}|\beta ; j, k\rangle, \quad\left|\mu^{j}\right|^{2}-\left|\nu^{j}\right|^{2}=1, \\
|\beta ; j, k\rangle & =\mathcal{N}^{-1}(j, k) \sum_{n=0}^{\infty} c_{n}(j, k)|n j+k\rangle, \quad k<j,
\end{aligned}
$$

where $\mathcal{N}(j, k)$ is the normalization constant. This time the equivalent MU method yields that these states satisfy equality for (the more general) Schrödinger uncertainty relation [10

$$
\left(\Delta X_{j}\right)^{2}\left(\Delta P_{j}\right)^{2} \geq \frac{1}{4}\left|\left\langle\left[X_{j}, P_{j}\right]\right\rangle\right|^{2}+\frac{1}{4}\left\langle\left\{\left(X_{j}-\bar{X}_{j}\right),\left(P_{j}-\bar{P}_{j}\right)\right\}\right\rangle^{2},
$$

$\{$,$\} being the anticommutator. By comparing Eqs. (5) and Eq. (65), one can then$ appreciate that the equation whose wave-function solution minimizes this uncertainty relation is of the form of Eq. (6), except that $B$ can now be complex and there is no restriction on $\Delta X / \Delta P$. (For the ordinary SS, this means the Gaussians need not have the width of the ground state and the squeeze can be complex.) 
Eq. (63) yields a three-term recursion relation [11 among the coefficients $c_{n+1}(j, k), c_{n}(j, k)$, and $c_{n-1}(j, k)$. It is

$$
\mu^{j}\left[\frac{[(n+1) j+k] !}{(n j+k) !}\right]^{1 / 2} c_{n+1}(j, k)+\nu^{j}\left[\frac{[n j+k] !}{[(n-1) j+k] !}\right]^{1 / 2} c_{n-1}(j, k)=\beta^{j} c_{n}(j, k),
$$

with boundary conditions determined by

$$
c_{1}(j, k)=\left(\frac{\beta}{\mu}\right)^{j}\left[\frac{k !}{(j+k) !}\right]^{1 / 2} c_{0}(j, k) .
$$

For $j>2$ this recursion relation has not been completely solved. But Nagel has studied it [4] from a Jacobi-matrix formulation. We proceed from his viewpoint.

Define

$$
B=\frac{\beta}{\mu}, \quad R=\left(\frac{\nu \mu}{\beta^{2}}\right)^{j}, \quad c_{n}(j, k)=\frac{b_{n}(j, k) B^{n j+k}}{[(n j+k) !]^{1 / 2}} .
$$

We now have the recursion relation

$$
b_{n+2}(j, k)=b_{n+1}(j, k)-R b_{n}(j, k) \frac{((n+1) j+k) !}{(n j+k) !}
$$

with boundary conditions (yet to be normalized) of

$$
b_{0}(j, k)=1, \quad b_{1}(j, k)=1 .
$$

Introduce the notation

$$
T_{n}(j, k)=\frac{(n j+k) !}{((n-1) j+k) !}=((n-1) j+k+1)_{j},
$$

where $(\alpha)_{N}=\Gamma(\alpha+N) / \Gamma(\alpha)$ is the Pochhammer symbol. The solutions for $b_{n}(j, k)$ are

$$
\begin{aligned}
b_{0}(j, k) & =b_{1}(j, k)=1, \\
b_{2}(j, k) & =1-R\left[T_{1}\right], \\
b_{3}(j, k) & =1-R\left[T_{1}+T_{2}\right], \\
b_{4}(j, k) & =1-R\left[T_{1}+T_{2}+T_{3}\right]+R^{2}\left[T_{1} T_{3}\right],
\end{aligned}
$$




$$
\begin{gathered}
b_{5}(j, k)=1-R\left[T_{1}+T_{2}+T_{3}+T_{4}\right]+R^{2}\left[T_{1} T_{3}+T_{1} T_{4}+T_{2} T_{4}\right], \\
b_{6}(j, k)=1-R\left[T_{1}+T_{2}+T_{3}+T_{4}+T_{5}\right]+R^{2}\left[T_{1} T_{3}+T_{1} T_{4}+T_{1} T_{5}\right. \\
\left.+T_{2} T_{4}+T_{2} T_{5}++T_{3} T_{5}\right]-R^{3}\left[T_{1} T_{3} T_{5}\right] .
\end{gathered}
$$

Higher- $n$ solutions continue with the same pattern.

The pattern is, first of all, a power series in $(-R)^{\left[\frac{n}{2}\right]}$. The factor multiplying $(-R)$ is the sum of all possible $T$ 's, up to $T_{n-1}$. The factor multiplying $(-R)^{2}$ is the sum of all possible products of two T's, that differ by order of at least two, up to the quantity $T_{n-1}$. The factor multiplying $(-R)^{3}$ is the sum of all products of three $T$ 's, each differing by order of at least two from the others, up to the quantity $T_{n-1}$, and so on. Symbolically, this is

$$
\begin{aligned}
& b_{n}(j, k)= \sum_{t=0}^{\left[\frac{n}{2}\right]}(-R)^{t} \sum_{\substack{\forall=1 \\
n-1}}^{t} \prod_{i=1}^{t} T_{v_{i}}(j, k), \\
& 1 \leq v_{1} \leq v_{2}-2 \leq \ldots \leq v_{n-2}-2 \leq v_{n-1} \leq n-1 .
\end{aligned}
$$

Using these b's,

$$
\begin{gathered}
|\beta ; j, k\rangle=\mathcal{N}^{-1}(j, k) \sum_{n=0}^{\infty} b_{n}(j, k) \frac{B^{n j+k}}{[(n j+k) !]^{1 / 2}}|n j+k\rangle, \\
\mathcal{N}^{2}(j, k)=\sum_{n=0}^{\infty} \frac{\left(B^{*} B\right)^{n j+k}}{[(n j+k) !]} b_{n}(j, k)^{*} b_{n}(j, k) .
\end{gathered}
$$

Converting to the number-state wave functions

$$
\psi_{n}(x)=\frac{\exp \left[-\frac{1}{2} x^{2}\right] H_{n}(x)}{\left[\pi^{1 / 2} 2^{n} n !\right]^{1 / 2}}
$$

we have

$$
|\beta ; j, k\rangle \rightarrow \frac{\exp \left[-\frac{1}{2} x^{2}\right]}{\mathcal{N}(j, k) \pi^{1 / 4}} \sum_{n=0}^{\infty} b_{n}(j, k)\left(\frac{B}{2^{1 / 2}}\right)^{n j+k} \frac{H_{n j+k}(x)}{(n j+k) !},
$$

If the $b_{n}(j, k)$ 's were a power in $[f(j, k) R]^{[n / 2]}$ (which their values are suggestive of), the sum in Eq. (82) would be of the form of the generating function $G$ of Eq. (19). One would again have $j$ orthogonal wave functions $(k=0,1, \ldots, j-1)$, each 
containing $j$ Gaussians (differently-squeezed than before), distributed evenly around the phase-space circle.

Finally, as observed by Nagel 四, one can demonstrate normalizability of the states. Note that, for large $n$, the recursion relation (69) is dominated by

$$
b_{n+2}(j, k) \approx-R b_{n}(j, k) T_{n+1}(j, k) .
$$

Then, the even and odd coefficients are decoupled and the highest-order in $R$ contri-

bution is dominant. (This pattern is seen in the specific $b$ 's we gave above.) Taking for definiteness the even- $n$ case, use Stirling's approximation to evaluate $T_{n+1}(j, k)$. One ends up with an exponent of a sum which one changes to an exponent of an integral. When one is finished evaluating, one finds

$$
\mathcal{N}^{2}(j, k) \rightarrow \text { Const. } \sum_{v=0}^{\infty}\left(\frac{\left|\nu^{2}\right|^{j}}{\left|\mu^{2}\right|^{j}}\right)^{v / 2} .
$$

From Eq. (63), this is a convergent geometric series. (Similarly for the odd- $n$ case.)

\section{Special-Case Solutions for the LO/MU Squeezed States.}

We start by considering the special case $(j, k)=(1,0)$. Here, the LO/MU-SS are identical to the DO-SS of Eqs. (54)-(56):

$$
\begin{gathered}
\mu=\cosh r, \quad \nu=-e^{i \phi} \sinh r, \quad \beta=\left[(\mu+\nu) x_{0}+i(\mu-\nu) p_{0}\right] / \sqrt{2}, \\
|\beta ; 1,0\rangle \rightarrow \psi_{s s}(x)=\left[\frac{(\mu+\nu)}{\pi^{1 / 2}(\mu-\nu)}\right]^{1 / 2} \exp \left[-\frac{\left(x-x_{0}\right)^{2}}{2} \frac{(\mu+\nu)}{(\mu-\nu)}+i p_{0} x\right] .
\end{gathered}
$$

The decomposition into number states is straight forward, and well known. However, it is enlightening to show how it fits into our general scheme. With the aid of Eqs. (54)-(56) the $c_{n}$ 's can be obtained from $c_{n}=\left(\psi_{n}, \psi_{s s}\right)$. This yields the $b_{n}(1,0)$ 's, which are

$$
b_{n}(1,0)=\left(\frac{R}{2}\right)^{n / 2} H_{n}\left[(2 R)^{-1 / 2}\right]=R^{n / 2} H e_{n}\left[R^{-1 / 2}\right]
$$




$$
\begin{aligned}
& =\sum_{t=0}^{\left[\frac{n}{2}\right]}(-R)^{t}\left[\frac{n !}{2^{t}(t !)[(n-2 t) !]}\right] \\
& =\left(-\frac{R}{2}\right)^{\left[\frac{n}{2}\right]} \frac{n !}{\left[\frac{n}{2}\right] !}{ }_{1} F_{1}\left(-\left[\frac{n}{2}\right] ; \frac{2+(-1)^{n+1}}{2} ; \frac{1}{2 R}\right),
\end{aligned}
$$

where ${ }_{1} F_{1}$ is the confluent hypergeometric function. This agrees with the special $(j, k)=(1,0)$ examples of our formulae (72)-(77). Further, putting the first equality of Eq. (87) and $T_{n}(1,0)=n$ back into the recursion relation (69) yields the standard Hermite polynomial recursion relation,

$$
H_{n+1}(x)=2 x H_{n}(x)-2 n H_{n-1}(x) .
$$

For $(j, k)=(2,0)$ and $(2,1)$, we have the ladder-operator even- and odd-squeezed states. The wave-function solutions are confluent hypergeometric functions 国, 8, 12 , [13, [14]:

$$
\begin{aligned}
& \psi_{(2,0) s s}(x)=N_{(2,0)} \exp \left[-\frac{x^{2}}{2}\left(U+\sqrt{U^{2}-1}\right)\right] \\
& \times{ }_{1} F_{1}\left(\left[\frac{1}{4}+\frac{B}{2 \sqrt{U^{2}-1}}\right] ; \frac{1}{2} ; x^{2} \sqrt{U^{2}-1}\right), \\
& \psi_{(2,1) s s}(x)=N_{(2,1)} x \exp \left[-\frac{x^{2}}{2}\left(U+\sqrt{U^{2}-1}\right)\right] \\
& \times{ }_{1} F_{1}\left(\left[\frac{3}{4}+\frac{B}{2 \sqrt{U^{2}-1}}\right] ; \frac{3}{2} ; x^{2} \sqrt{U^{2}-1}\right), \\
& U=\frac{\left(\mu^{2}-\nu^{2}\right)}{\left(\mu^{2}+\nu^{2}\right)}, \quad B=\frac{\beta^{2}}{\left(\mu^{2}+\nu^{2}\right)} .
\end{aligned}
$$

The shapes of the states so produced resemble the "effective" DO-SS with their Gaussians $[8]$.

Nagel [4] discussed the decomposition into the number states $|2 n+k\rangle, k=(0,1)$. One obtains that the $c_{n}(2, k)$ are proportional to Pollaczek polynomials [4],

$$
P_{n}(x, \delta) \equiv i^{n} \sqrt{(2 \delta)_{n} / n !}{ }_{2} F_{1}(-n,(\delta+i x) ; 2 \delta ; 2) .
$$


When put in our notation, the results are

$$
\begin{aligned}
& b_{n}(2,0)=i^{n}\left(\frac{1}{2}\right)_{n} 2^{n} R^{n / 2}{ }_{2} F_{1}\left(-n,\left(\frac{1}{4}+\frac{i}{4 R^{1 / 2}}\right) ; \frac{1}{2} ; 2\right), \\
& b_{n}(2,1)=i^{n}\left(\frac{3}{2}\right)_{n} 2^{n} R^{n / 2}{ }_{2} F_{1}\left(-n,\left(\frac{3}{4}+\frac{i}{4 R^{1 / 2}}\right) ; \frac{3}{2} ; 2\right) .
\end{aligned}
$$

Putting these $b_{n}$ 's into Eq. (69) reduces it to the Gauss contiguous relation

$0=(c-a)_{2} F_{1}(a-1, b ; c ; z)+(2 a-c-a z+b z)_{2} F_{1}(a, b ; c ; z)+a(z-1)_{2} F_{1}(a+1, b ; c ; z)$.

\section{$7 \quad$ Experimental Realizations}

The simplest higher-power coherent states, the even- and odd-coherent states, are commonly called "Schrödinger Cat States," since they are the mathematical realizations of Schrödinger's gedanken cat that is simultaneously dead and alive. Although this "cat" was for decades a matter of heated epistemological debate, with the advent of modern quantum optics such states have now been demonstrated in two different physical systems.

Wineland's group [15] was able, with much effort, to entangle ${ }^{9} \mathrm{Be}^{+}$ions in a trap, producing even- and odd- coherent states. The method starts with the ion in its vibrational ground state. Then, with a $\pi / 2$ laser pulse the hyperfine levels are mixed. A different kicking laser excites only the upper hyperfine level into an energetic coherent state. Both sets of internal states are then swapped by a $\pi / 2$ laser pulse. Finally, the motionless component, is excited by by a new kicking pulse, yielding a mixed state. Mind you, in general they produce two wave packets with differing relative phases. The relative phases must be adjusted to 0 and $\pi$ to yield the orthonormal even- and odd-states.

The second system, studied by Haroche's group [16], a rubidium atom is prepared in a mixture of circular $n=50$ and $n=51$ Rydberg states. This atom is then sent 
through a high-Q cavity with a few coherently produced photons in it. The traversing atom's two states shift the phases of the photons differently, thus producing entangled field/atom states. By re-mixing the the Rydberg states after the atom leaves the cavity, EPR states can be produced. [See Ref. [17] for a popular account of both these systems.]

Both systems appear amenable, in principle, to extensions producing 3-power or 4-power coherent states. However, this would be complicated to actually achieve in practice. Even so, such extensions would be of interest. For example, as Gerry has emphasized [18], the 3-power states arise in models of trapping. In the two-channel model of Jyotsna and Agarwal [19], there are certain trapping states that involve CS that are eigenstates of $a^{3}$. These are explicitly the 3-power coherent states.

Additionally, all these states could be, again in principle, squeezed [20, 21].

\section{Acknowledgements}

We thank Bengt Nagel for illuminating communications. We also thank George A. Baker, Jr. for discussions on normalizability. MMN acknowledges the support of the United States Department of Energy and the Alexander von Humboldt Foundation. DRT acknowledges a grant from the Natural Sciences and Engineering Research Council of Canada.

\section{References}

[1] V. V. Dodonov, I. A. Malkin, and V. I. Man'ko, Physica 72 (1974) 597.

[2] V. Bužek, I. Jex, and Tran Quang, J. Mod. Optics 37 (1990) 159; I. Jex and V. Bužek, ibid. 40 (1993) 771; I. Jex, P. Törmä, and S. Stenholm, ibid. 42 (1995) 1377. 
[3] M. M. Nieto and D. R. Truax, Phys. Lett. A 208 (1995) 8. Also see F. M. Fernández, ibid. 237 (1998) 189; M. M. Nieto and D. R. Truax, ibid 237 (1998) 192.

[4] B. Nagel, in: Proceedings of the Fifth International Conference on Squeezed States and Uncertainty Relations, NASA Conference Publication NASA/CP1998-206855, ed. D. Han, J. Janszky, Y. S. Kim, and V. I. Man'ko (NASA, Washington, DC, 1998), p. 43. Eprint quant-ph/9711028.

[5] M. M. Nieto and L. M. Simmons, Jr., Phys. Rev. D 20 (1979) 1321.

[6] M. M. Nieto and D. R. Truax, Fortschr. Phys. 45 (1997) 145. Also see R. A. Fisher, M. M. Nieto, and V. D. Sandberg, Phys. Rev. D 29 (1984) 1107; S. L. Braunstein and B. L. McLachlan, Phys. Rev. A 35 (1987) 1639; and Ref. [四].

[7] R. L. de Matos Filho and W. Vogel, Phys. Rev. Lett. 76 (1996) 608.

[8] M. M. Nieto, Phys. Lett. A 219 (1996) 180.

[9] M. M. Nieto, Phys. Lett. A 229 (1997) 135.

[10] E. Schrödinger, Sitzungber. Preuss. Akad. Wiss. (1930) 296.

[11] C. M. Bender and S. A. Orszag, Advanced Mathematical Methods for Scientists and Engineers (McGraw-Hill, New York, 1978), Sec. 2.3.

[12] M. Hillery, Phys. Rev. A 36 (1987) 3796; J. A. Bergau, M. Hillery, and D. Yu, Phys. Rev. A 43 (1991) 515; G. Satya Prakash and G. S. Agarwal, Phys. Rev. A 50 (1994) 4258; P. Shanta, S. Chaturvedi, and V. Srinivasan, Mod. Phys. Lett. A 11 (1996) 2381; P. Marian, Phys. Rev. A 55 (1997) 3051.

[13] M. M. Nieto and D. R. Truax, Phys. Rev. Lett. 71 (1993) 2843. 
[14] B. Nagel, in: Modern Group Theoretical Methods in Physics, ed. J. Bertrand, et al. (Kluwer, NL, 1995), p. 221. Eprint quant-ph/9711018.

[15] C. Monroe, D. M. Meekhof, B. E. King, and D. J. Wineland, Science 272 (1996) 1131.

[16] M. Brune, E. Hagley, J. Dreyer, X. Maitre, A. Maali, C. Wunderlich, J. M. Raimond, and S. Haroche, Phys. Rev. Lett. 77 (1996) 4887.

[17] S. Haroche, Physics Today 51 (1998) No. 7, 36.

[18] C. C. Gerry, private communication.

[19] I. V. Jyotsna and G. S. Agarwal, Phys. Rev. A 50 (1993) 1770.

[20] D. M. Meekhof, C. Monroe, B. E. King, W. M. Itano, and D. J. Wineland, Phys. Rev. Lett. 76 (1996) 1796; D. Liebfried, D. M. Meekhof, B. E. King, C. Monroe, W. M. Itano, and D. J. Wineland, Phys. Rev. Lett. 77 (1996) 4281.

[21] See, e.g., the special issue Appl. Phys. B 55 (1992) No. 3. 